\title{
Geleitwort von Hedwig François-Kettner (1. Auflage)
}

Namhafte Experten im Gesundheitswesen sehen in einer sicheren Patientenversorgung den interdisziplinären und multiprofessionellen Auftrag zu einer lückenlosen Zusammenarbeit über alle Grenzen der sektoralen Betreuung hinweg. Das bedeutet, dass jede Berufsgruppe den Blick auf den Patienten richtet und seine sichere Versorgung im Fokus behält (auch nach Verlassen seines unmittelbaren Handlungsfeldes). Gleichzeitig bedeutet es auch unbedingt, systemseitige Schwächen zu erkennen, sie nachhaltig zu verändern, sie zu überwinden und das mit allen Partnern gemeinsam. Die Erfahrungen, die wir machen, müssen durch Forschungen gestützt werden, um nach sachgerechten Analysen die richtigen Schritte zur Prävention und Versorgung vorzunehmen.

Dieter Hart beschrieb es sehr treffend in seinem Aufsatz in der Zeitschrift Medizinrecht 2012. Ich zitiere:

1. Patientensicherheit ist ein System- und Organisationsproblem (Risiko- und Organisationsforschung) und nicht nur das eines individuellen Fehlverhaltens;

2. es ist wichtig zu wissen, was die Aktivitäten der Sicherheitsinstitutionen, insbesondere bei den Organisationen im Gesundheitswesen, Krankenhäusern, Pflegeheimen und Arztpraxen, bewirken, also Entwicklungen zu evaluieren, um Nutzen und Kosten von vermutlich sicherheitsfördernden Maßnahmen bewerten zu können (Versorgungsforschung) und

3. es bedarf konzentrierter und konzertierter Anstrengungen aller Beteiligter, auch der Patienten, um Patientensicherheitsziele zu präzisieren und zu erreichen.

Patientensicherheit wird in diesem Buch aus allen Blickwinkeln beleuchtet. Es wird deutlich, wie komplex das Thema ausfällt und das jede(r)Einzelne „zuständig“ ist, daran mitzuwirken. Neben der Sicht auf das Individuum und den systemseitigen Rahmen in Deutschland, die beide strukturgebend sind, werden aus verschiedenen Aspekten heraus prozesshafte Abläufe und methodisches Vorgehen beleuchtet. Die Verantwortung des Managements in jeder Institution und die Interaktion des einzelnen Akteurs werden sichtbar, sind evident und bedingen sich. Technische Systeme mit vernetzter elektronischer Unterstützung könnten dabei m. E. zu deutlich größerer Patientensicherheit beitragen.

1 Hart D. Patientensicherheit, Fehlermanagement, Arzthaftungsrecht - zugleich ein Beitrag zur rechtlichen Bedeutung von Empfehlungen. MedR. 2012;30(1):1-15. 
Daher ist der Titel des vorliegenden Werks „Patientensicherheitsmanagement“ sachgerecht gewählt. Er verdeutlicht, dass gezielte Managementmethoden notwendig sind - zur Förderung der Patientensicherheit.

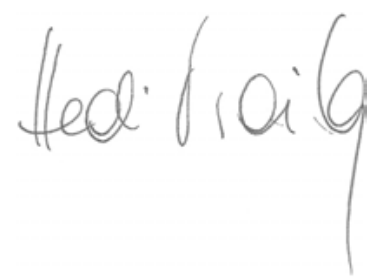

Hedwig François-Kettner

Vorsitzende des Aktionsbündnisses Patientensicherheit 